\title{
Effect of verbal coach encouragement on rating perceived exertion and mood state during small sided games in professional soccer players
}

\begin{abstract}
The purpose of this study was to compare the effects of coach encouragement during small-sided games (SSG) on mood state and rating of perceived exertion (RPE) in professionals' soccer players. Twenty soccer players (mean \pm SD: age: $23 \pm 0.9$ years; height: $1.80 \pm 0.09 \mathrm{~m}$; weight: $74.05 \pm 7.99 \mathrm{~kg}$, \%Fat: $11.05 \pm 3.1 \%)$ ) took part in this study. Testing of players was conducted on separate days (each training session: $25-$ min: $4 \times 4$-min work with 3-min of passive recovery in-between) under two conditions with and without verbal encouragement during 4 vs. 4 SSG on a $24 \times 35-m$ pitch size. Mood state was measured using the profile of mood state questionnaire (POMS). The players' heart rate (HR) was continuously measured, whereas ratings of perceived exertion (RPE) were collected after each bouts of SSG. RPE during SSGE and percent of HRmax (\%HRmax) were higher in comparison than SSGNE $(p<0.01)$. The SSGNE compared with SSGE resulted in: an increased total mood disturbance $(\mathrm{p}<0.01)$, tension $(p<0.05)$ and a decreased vigor $(p<0.01)$. SSGNE produced a mood disturbance while SSG ensured mood balance and high intensity. Our findings highlight the efficiency of verbal coach encouragement in games intensity and the psychological state.
\end{abstract}

Volume I Issue 4 - 2017

\author{
Okba Selmi, ${ }^{1,2}$ Anissa Bouassida' \\ 'Department of Sportive Performance and Physical \\ Rehabilitation, University of Jendouba, Tunisia \\ ${ }^{2}$ Faculty of Sciences of Bizerte, University of Carthage, Tunisia
}

Correspondence: Okba Selmi, Department of Sportive Performance and Physical Rehabilitation, High Institute of Sports and Physical Education of Kef, University of Jendouba, Tunisia, Tel 0021693809606,Email okbaselmii@yahoo.fr

Received: September 09, 2017| Published: October 04, 2017

Keywords: Coach, players, exercise intensity, motivation, physiological state

Abbreviations: RPE, rating of perceived exertion; SSG, small-sided games; POMS, profile of mood state questionnaire; HR, heart rate

\section{Introduction}

Usually, most coaches used training exercises without the ball to develop soccer players' physical conditioning. ${ }^{1}$ More recently, scientific research showed that one can maintain or develop the physical capacity while soliciting concomitantly the technical and tactical parameters in the same training session by using soccer specific training exercises, which usually include game..$^{2-4}$ Furthermore, apart from physiological benefits, SSGs may result in better motivation and developed more positive attitudes. ${ }^{5}$ The most evident distinction between SSG and other training methods is the presence of the ball, which imposes a specific training soliciting the physical, technical and tactical enhancement with high player enjoyment. ${ }^{6}$ It has recently been proposed that SSG should be positive for physiological, technical, tactical and psychological enhancement ${ }^{7}$ and may be more effective than other training modality in plummeting the possibility of sensitive consequences associated with obtaining lack of mood state. ${ }^{8}$ Indeed, Los Arcos et al. ${ }^{7}$ reported that the participation in the SSG can develop both physiological responses and psychological of players. In the same perspective Selmi et al. ${ }^{5}$ indicated that SSG can be used as a specific type of training for elite soccer players allowing soccer-specifically intensively soliciting their physiological responses while sparing their mood state. During SSGs, the verbal coach encouragement is considered as external motivation provided by coaches and physical trainer. It has been shown to attain high commitment, positive behavior and training adherence for instance. ${ }^{9}$ To the best of our knowledge no studies examined the effects of the verbal coach encouragement on mood state. Indeed, the verbal coach encouragement would directly impact the physical intensity (such as rating of perceived exertion (RPE)) of players' activity. ${ }^{10}$ Other studies reported the importance of the verbal coach encouragement on the game intensity and the player's physiological response to SSG. Verbal encouragement is considered as external motivation provided by technical staff. It has been shown to attain high commitment, positive behavior and training adherence for instance, during high-intensity training. ${ }^{9}$ However, Rampinini et al. ${ }^{4}$ studied the effect of coaches' verbal encouragement in soccer players' on physiological responses during different modality of SSG: 3 vs. 3, 4 vs. 4, 5 vs. 5 and 6 vs. 6 players on small, medium and large pitches area. Researchers indicated that the heart rate (HR), blood lactate concentration (La) and RPE responses to SSG were significantly higher SSG with verbal coach encouragement in comparison with no-verbal coach encouragement during all SSG formats. Carrying out these various findings, the aim of this study is to compare the mood state, the RPE and \%HRmax between SSG with verbal coach encouragement (SSGE) and without verbal coach encouragement (SSGNE) in soccer players. We hypothesized that SSGE would produce high-RPE and HRmax and better mood state compared to SSGNE.

\section{Materials and methods}

\section{Participants}

Twenty players among the same professional soccer team competing in the first national league in Tunisia (high-level) took part in the study (age: $23 \pm 0.9$ years; height: $1.80 \pm 0.09 \mathrm{~m}$; weight: $74.05 \pm 7.99 \mathrm{~kg}, \%$ Fat: $11.05 \pm 3.1 \%$ ). All players had a minimum of 11.5 years of experience in competitive soccer. On average, the players trained 6-7 times per week $(10.1 \pm 1.54$ hours of training/ week). Goalkeepers were excluded from the investigation as they did not participate in the same physical training program. The subjects 
were familiar with all protocol procedures as part of their regular performance assessment program and were required to keep their eating and drinking habits constant during the test period. All subjects received written and verbal explanations on the study informing them of all risks and benefits associated with participation. Before data collection, written informed consent was obtained. This study was approved by the Research Ethics Committee of High Institute of Sports and Physical Education of Kef and was consistent with the institutional ethical requirements for human experimentation in accordance with the Declaration of Helsinki.

\section{Procedure}

Data collection was divided into three stages on separate days. The players' first visit was to assess their anthropometric characteristics and the maximal heart rate (HRmax) which was determined through the VAMEVAL test. ${ }^{11}$ The experimental sessions were performed on the second and third visits (i.e., 5 vs. 5 SSGNE HIIT and 5 vs. 5 SSGE, respectively), in which studied variables were collected before (i.e., mood state), during (i.e., HR) and after (i.e., mood state and RPE) each session. The experimental session's tests were performed during the competitive period in the same training field (i.e., natural grass) and at the same time of the day between 9 and 10 am in order to limit the effects of the circadian variations on the measured variables, particularly on heart rate (HR) measurements. ${ }^{12}$ Each protocol was preceded by a 20-min standardized warm-up, which consisted of low intensity running, striding and stretching. Players were allowed to consume available drinks during the recovery periods.

\section{Measures}

\section{Anthropometric measurements}

Standing height and body mass were measured using standard techniques with a variability of $0.2 \mathrm{~kg}$ and $5 \mathrm{~mm}$, respectively. Height was measured to the nearest $0.1 \mathrm{~cm}$ and body mass was measured to the nearest $0.1 \mathrm{~kg}$ with a digital scale (OHAUS, Florhman Park, NJ). Skin fold thickness was determined in triplicate at four standard sites (biceps, triceps, sub scapular and sup railiac), using a calibrated Harpenden caliper (Holtain Instruments, Crosswell, Pembroeshire, UK). Body density was estimated according to the equations of Durnin \& Womersley ${ }^{13}$ for men aged 20-65 years and body fat was calculated by using Siri's equation. ${ }^{14}$

\section{Rating perceived exertion}

Internal intensity were recorded immediately after the SSGs using the RPE scale (Borg CR-10 scale), as proposed earlier. ${ }^{15}$ Each player's RPE was collected to ensure that the perceived effort was referred to the small-sided game training only.

\section{Small-Sided games training}

SSGE and SSGNE were played in random order at two day intervals without a goalkeeper. Each 4 vs. 4 SSG situations were performed on a playing surface of $30 \mathrm{~m}$ long and $20 \mathrm{~m}$ wide and had an overall duration of $25 \mathrm{~min}$ and it consisted of 4 bouts of $4 \mathrm{~min}$ duration with 3 min passive recovery between exercise bouts. The pitch size and duration of 4 vs. 4 SSG were strictly controlled and have been implemented by other researchers. ${ }^{2,3}$ The number of ball touches authorized per individual possession was fixed at two ball touch. The participants were asked to perform a maximum effort during the games and to maintain the possession of the ball for the longest time possible. During the SSGE, trainer moves around the perimeter of the pitch to encourage the participants and to provide new balls when necessary to allow continuity of the play during the playing sessions. During the SSGNE, trainer stands next filed and provide new balls when necessary to allow continuity of the play during the playing sessions and no verbal encouragement. The participants were well familiarized with tow protocols.

\section{The profile of mood state (POMS)}

The POMS is a 65-item instrument that measures mood states on a 5-level adjectival scale from 0 to 4 : not at all, a little, moderately, quite a bit and extremely. ${ }^{16}$ The POMS measures scores along 6 mood states: tension-anxiety, depression-dejection, anger-hostility, fatigueinertia, confusion-bewilderment and vigor-activity. The six subscales of POMS can be combined into a Total Mood Disturbance (TMD) score by summing the $\mathrm{T}$ scores for the five negative mood subscales and subtracting the $\mathrm{T}$ score for positive mood state and adding a constant of 100 in order to prevent negative numbers

$\mathrm{TMD}=\{($ Anger + Confusion + Depression + Fatigue + Tension $)$ - Vigor +100$\}$

\section{VAMEVAL test}

In order to obtain individual MAS and HRmax, all participants completed the VAMEVAL test. ${ }^{11}$ The test was performed on a 200 $\mathrm{m}$ outdoor running track. Players ran guided by ten cones placed every $20-\mathrm{m}$ at specific sites of the field following a pre-programmed auditory signal (i.e., beep). The speed of the test was initially set at $8 \mathrm{~km} . \mathrm{h}-1$ and increased subsequently by $0.5 \mathrm{~km} . \mathrm{h}-1$ every minute until exhaustion. ${ }^{11}$ Participants adjusted their running speed to the cones placed at $20-\mathrm{m}$ intervals. The test was stopped when a subject could no longer maintain the required running speed dictated by the beep for two consecutive occasions or felt that he could not complete the stage. The velocity of the last 1-min stage for each subject was retained as the player's MAS. The HR was measured and stored using a Polar Team Sport System (Polar-Electro OY, Kempele, Finland). The highest average value over 5 seconds during the test was recorded as VamevalHRmax.

\section{Physiological measurements}

HRmax was determined by the completion of the VAMEVAL test ${ }^{11}$ in which the highest values of heart rate (HR) reached during the test corresponded to the HRmax. HR was continuously monitored throughout the training intervention by HR monitors (Polar Team Sport System, Polar-Electro OY, Kempele, Finland), recorded every 5 -s intervals. The mean HR for each bout was calculated to obtain the means of the 4 bouts of training (HRmean). The exercise HR expressed in \%HRmax was calculated by the following formula for each form of training

$\%$ HRmax $=($ HRmean $/$ VamevalHRmax $) \times 100$

\section{Data analysis}

Statistical analyses were performed using SPSS version 20 for Windows (SPSS Inc, Chicago, Il, USA). Data in text are presented as mean \pm standard deviation $( \pm \mathrm{SD})$. The distribution of each variable was examined with the Kolmogorov-Smirnov normality test. Paired t-tests were used to compare both training methods for PACES. A two-way analyses of variance (ANOVA) with repeated measures was used to determine the effect of the condition (SSGE or SSGNE), the 
effect of bouts ( 4 bouts) and their interaction (condition $\times$ time) on RPE and HR values. When a significant interaction effect was found, the analysis was completed with a post-hoc Bonferroni test. Effect size (ES) (Cohen's d) ${ }^{17}$ was calculated to determine the practical difference between SSGE and SSGNE. Effect sizes values $<0.2,0.2$ $0.6,0.6-1.2,1.2-2.0,2.0-4.0$ and 2.0-4.0, were considered as trivial, small, moderate, large and very large, respectively. ${ }^{18}$ The level of statistical significance was set at $\mathrm{p}<0.05$.

\section{Results}

Paired t-tests were used to compare the effects of the two training session on RPE and \%HRmax. RPE and \%HRmax were significantly higher in SSGE compared to SSGNE $(\mathrm{P}<0.001, \mathrm{ES}=1.22 ; \mathrm{P}<0.001$, $\mathrm{ES}=1.31$, respectively) (Figure 1) Concerning the POMS scores, no significant main effects of condition and time and no significant interaction on the scores of fatigue, anger, confusion and depression were observed (Table 1). However, there was a significant main effect of condition on tension, vigor and total mood disturbance (TMD), a significant main effect of time on tension, vigor and TMD (1) and a significant interaction effect on tension, vigor and TMD (Table 2). Post-hoc analyses indicated that tension and TMD increased significantly in SSGNE compared with the SSGE and vigor decreased significantly in SSGNE compared with the SSGE.

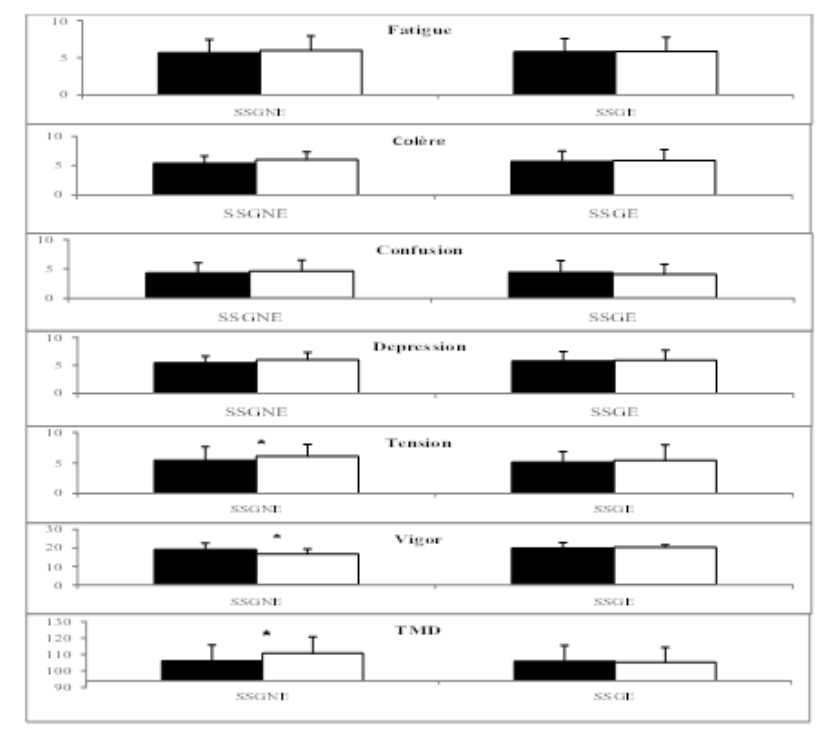

Figure I Mean POMS scores for both small-sided games with verbal coach encouragement (SSGE) and without verbal coach encouragement (SSGNE) collected before (pre-training) and after (post-training) each session.

Table I Comparison of \%HRmax and RPE between small-sided games with verbal coach encouragement (SSGE) and without verbal coach encouragement (SSGNE)

\begin{tabular}{llllll}
\hline Variables & Training method & Mean \pm SD & $\mathbf{p}$ & $|\mathbf{d}|$ & ES \\
\hline \%HRmax (beat.min-I) & SSGE & $85.39 \pm 3.31$ & 0.01 & $3.38^{*}$ & 0.81 \\
& SSGNE & $82.01 \pm 3.62$ & & & \\
RPE & SSGE & $6.61 \pm 0.91$ & 0.003 & $0.73 * *$ & 0.92 \\
& SSGNE & $5.88 \pm 0.96$ & & &
\end{tabular}

Note \%HRmax, Percentage of Maximal Heart Rate; RPE, Rating of Perceived Exertion; |d|, Difference between SSG and HIIT; ES, Size Effect

Table 2 Results of the ANOVA with $2 \times 2$ repeated measures [Condition ((small-sided games with verbal coach encouragement (SSGE) and without verbal coach encouragement (SSGNE)) $\times$ Time (pre- and post-training)]

\begin{tabular}{|c|c|c|c|c|c|c|}
\hline \multirow[t]{2}{*}{ Main effects variables } & \multicolumn{2}{|c|}{ Training method } & \multicolumn{2}{|l|}{ Effort } & \multicolumn{2}{|c|}{ Interaction } \\
\hline & $F(1,15)$ & $\eta 2$ & $F(1,15)$ & $\eta 2$ & $F(1,15)$ & $\eta 2$ \\
\hline Tension & $7.5^{* *}$ & $0.44 * *$ & $6.8 I^{*}$ & $0.29 *$ & $5.02^{*}$ & $0.30 *$ \\
\hline Anger & 0.11 & 0.01 & 0.63 & 0.04 & 0.51 & 0.03 \\
\hline Confusion & 1.09 & 0.08 & 0.64 & 0.04 & 0.24 & 0.02 \\
\hline Depression & 0.55 & 0.04 & 0.5 & 0.03 & 0.15 & 0.01 \\
\hline Fatigue & 3.84 & 0.2 & 3.99 & $0.21 * * *$ & 3.12 & 0.18 \\
\hline Vigor & 8. I** & $0.40 * *$ & $9.68 * *$ & $0.42 * *$ & $19.97 * * *$ & $0.59 * * *$ \\
\hline TMD & $|3.2| * * *$ & $0.47 * * *$ & $21.01 * * *$ & $0.60 * * *$ & $30.01 * * *$ & $0.70 * * *$ \\
\hline
\end{tabular}

Note TMD, Total Mood Disturbance. ${ }^{*} \mathrm{p}<.05,{ }^{*} \mathrm{p}<.01, * * \mathrm{p}<.001$

\section{Discussion}

The aim of the present study was to $T$ to determine the effect of verbal coach encouragement during 5 vs. 5 SSG on the mood state, the RPE-SSG and \%HRmax in professional soccer players. The present study showed that:
I. RPE-SSG and \%HRmax were higher in SSGE compared to SSGNE

II. SSGE resulted in positive mood compared to SSSGNE. The results of the study confirmed the hypotheses that during the SSGE, \%HR max and RPE would remain significantly higher. 
The results of the study confirmed the hypotheses that during the SSGE, \%HR max and RPE would remain significantly higher than during the SSGNE. This indicates that players participating in SSGE worked at high intensity. ${ }^{10}$ The 5 vs. $5 \mathrm{SSG}$, as performed here, would therefore be considered as an intense exercise that could result in training adaptations if used repeatedly in a training program. ${ }^{5,6}$ Verbal coach encouragement is referred by several studies as one of the factors that influence the player's physiological response and intensity to $\mathrm{SSG}^{4-6}$

Indeed, Hill-hass et al. ${ }^{6}$ showed that In SSG, coach encouragement has also been suggested to have an influence on soccer specific training intensity. In the same context, Rampinini et al. ${ }^{4}$ showed that encouragement has caused the highest values in terms of heart rate and RPE during SSG. 4 vs. 4 SSG by changing the pitch dimensions with or without encouragement gave the following values for $\%$ HRmax (small: $88.7 \pm 2.0,86.5 \pm 3.4 \%$; medium: $89.4 \pm 1.8,86.7 \pm 3.0 \%$; large: $89.7 \pm 1.8,87.2 \pm 2.8 \%$ ) and for RPE (small: $7.6 \pm 0.5,6.3 \pm 0.5$; medium: $7.9 \pm 0.5,6.6 \pm 0.6$; large: $8.10 .5,6.8 \pm 0.5)$ respectively. ${ }^{4}$ Another important finding concerned the comparison of mood state (POMS) between SSGE SSGNE. Many studies highlighted the importance of the POMS questionnaire in evaluating mental fatigue in players..$^{19,20}$ The results of the present study indicated that SSGNE caused a significant increase in total mood disturbance (TMD), tension and a significant decrease in vigor unlike the SSGE which resulted in no-change. This indicates that SSGNE elicits an increase in negative statements and decreased positive mood. These results demonstrate that mood state is negatively influenced by the absence of encouragement variables. Mood balance during SSGE can be explained by the opportunity to repeatedly touch the ball and encouragement during exercise due to the increase the motivation factors. Based on the above, it can be suggested that SSGNE causes displeasure and mental fatigue among players compared with SSGE. ${ }^{7,21}$ It has been suggested that mood disturbance is associated with unpleasant sensations. ${ }^{5}$ In this context, Chitwood et al. ${ }^{22}$ indicated that encouragement during training exercise is expected to increase motivation and improve positive behavior.

Although this study found mood disturbance in SSGNE compared to a higher stability of the mood state in SSGE, several limitations must be taken into account when interpreting the results. Firstly, the number of participants in this protocol is reduced $(n=20)$ due to the difficulty to recruit a homogeneous large number of participants in the same conditions. Secondly, only one format of SSG was used, modifying the player numbers, pitch size, number of bouts and recovery periods, using goals and goalkeepers may have provided further insight of the effect of altering recovery duration on different SSG formats. Finally, it would be interesting to associate these responses with hormonal variables because these parameters are important marker of psychosomatic status. These factors should be controlled for in future researches.

\section{Conclusion}

To the best knowledge of the authors, this study is the first to address SSG in soccer to determine the effect of verbal coach encouragement on mood state. This should be addressed in professional soccer players. Henceforth the verbal coach encouragement can be used as a determining factor in the training sessions for players allowing soccer to intensely soliciting their physical commitment with a mood balance. SSGE sessions induced higher RPE and \%HRmax, in contrast, SSGNE produced a mood disturbance while SSGE ensured mood stability. Coaches should also give importance to the social factors that perceived coaches' behavior can be essential to increase the perceived competence and intrinsic motivation levels of players during related practice game.

\section{Acknowledgements}

None.

\section{Conflict of interest}

Author declares there is no conflict of interest in publishing the article.

\section{References}

1. Little T, Williams AG. Measures of exercise intensity during soccer training drills with professional soccer players. J Strength Cond Res. 2007;21(2):367-371.

2. Dellal A, Jannault R, Segovia LM, et al. Influence of the numbers of players in the heart rate responses of youth soccer players within 2 vs. 2 , 3 vs. 3 and 4 vs. 4 small-sided games. J Hum Kinet. 2011;28:107-114.

3. Haas HSV, Coutts AJ, Dawson BT, et al. Time-motion characteristics and physiological responses of small-sided games in elite youth players: the influence of player number and rule changes. J Strength Cond Res. 2010;24(8):2149-2156.

4. Rampinini E, Impellizzeri FM, Castagna C, et al. Factors influencing physiological responses to small-sided soccer games. J Sports Sci. 2007;25(6):659-666.

5. Selmi O, Haddad M, Majed L, et al. Soccer training: high-intensity interval training is mood disturbing while small sided games ensure mood balance. J Sports Med Phys Fit, Tunisia. 2017.

6. Haas HSV, Dawson B, Impellizzeri FM, et al. Physiology of small-sided games training in football. Sports Med. 2011;41(3):199-220.

7. Arcos AA, Juan SV, Juan M, et al. Effects of Small-Sided Games vs. Interval Training in Aerobic Fitness and Physical Enjoyment in Young Elite Soccer Players. PloS one. 2015;10(9):e0137224.

8. Álvarez MS, Balaguer I, Castillo I, et al. Coach autonomy support and quality of sport engagement in young soccer players. Span J Psychol. 2009;12(1):138-148.

9. Aguiar M, Botelho G, Lago C, et al. A review on the effects of soccer small-sided games. J Hum Kinet. 2012;33:103-113.

10. Dellal A, Chamari K, Pintus A, et al. Heart rate responses during smallsided games and short intermittent running training in elite soccer players: a comparative study. J Strength Cond Res. 2008;22(5):1449-1457.

11. Chtara M, Chamari K, Chaouachi M, et al. Effects of intra-session concurrent endurance and strength training sequence on aerobic performance and capacity. Brit J Sports Med. 2005;39(8):555-560.

12. Drust B, Waterhous J, Atkinson G, et al. Circadian rhythms in sports performance an update. Chronobiol Int. 2005;22(1):21-44.

13. Durnin J, Womersley. Body fat assessed from total body density and its estimation from skinfold thickness: measurements on 481 men and women aged from 16 to 72 years. Brit J of Nut. 1974;32(1):77-97.

14. Siri W. Body composition from fluid spaces and density: analysis of methods. Nutrition. 1993;9(5):480-491.

15. Foster C, Florhaug JA, Franklin J, et al. New approach to monitoring exercise training. J Strength Cond Res. 2001;15(1):109-115. 
16. Nair MDM, Droppleman LF, Lorr M. Edits manual for the profile of mood states. POMS; USA; 1984. p. 7.

17. Cohen J. The concepts of power analysis. Statistical power analysis for the behavioral sciences, Lawrence Erlbaum Associate Publishers, London; 1992. p. 1-17.

18. Hopkins W, Marshall S, Batterham A, et al. Progressive statistics for studies in sports medicine and exercise science. Med Sci in Sports Exer. 2009;41(1):3-13.

19. Morgan W, Brown D, Raglin J, et al. Psychological monitoring of overtraining and staleness. Brit J Sports Med. 1987;21(3):107-114.
20. Mashiko T, Umeda T, Nakaji S, et al. Position related analysis of the appearance of and relationship between post-match physical and mental fatigue in university rugby football players. Brit $J$ Sports Med. 2004;38(5):617-621.

21. Mavridis G, Kouli O, Rokka S. The impact of exercise intensity on mood state of participants in dance aerobics programs. Stud in Phys Cul and Tour. 2010;17(3):241-245.

22. Chitwood LF, Moffatt RJ, Burke K, et al. Encouragement during maximal exercise testing of type A and type B scorers. Percept Mot Skills. 1997;84(2):507-512. 